\title{
Composite $\mathrm{Fe}_{3} \mathrm{O}_{4} @ \mathrm{Au}$ Core-Shell Nanoparticle: Tunable and Enhancement of Optical Absorption Property
}

\section{ARTIT CHINGSUNGNOEN ${ }^{1}$, PANUWAT CHAIYACHATE ${ }^{2}$ and THANANCHAI DASR ${ }^{3 *}$}

\author{
${ }^{1}$ Technological Plasma Research Unit, Department of Physics, Faculty of Science, \\ Mahasarakham University, 44150, Thailand.

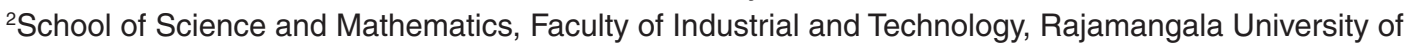 \\ Technology Isan Sakon Nakhon Campus, Sakon Nakhon, 47160, Thailand. \\ ${ }^{3}$ Faculty of Applied Science and Engineering, Khon Kaen University, Khon Kaen, 40002, Thailand. \\ "Corresponding author E-mail: thananchai_dasri@ hotmail.com \\ http://dx.doi.org/10.13005/ojc/330407
}

(Received: March 29, 2017; Accepted: May 03, 2017)

\begin{abstract}
Bifunctional nanomaterial $\mathrm{Fe}_{3} \mathrm{O}_{4} @ \mathrm{Au}$ core-shell is a kind of nanoparticle that includes magnetic iron oxide core with gold coated. It can be used in can achieve the controllable and tunable electromagnetic field enhancement and may open up exciting opportunities to engineer new materials used in biomedical applications. This research work aims at theoretical investigating the basic optical absorption property of $\mathrm{Fe}_{3} \mathrm{O}_{4} @ \mathrm{Au}$ core-shell with varying number particles, inter-particle distance, and direction of incident light using the discrete dipole approximation method (DDA) in the wavelength of visible to near-infrared ranges of the electromagnetic wave spectrum. The results were observed that these parameters can be enhanced optical light absorption and tuned the LSPR peak position. The obtained results might be used for basic fabricating the more performance specific applications like a drug in life science.
\end{abstract}

Keywords: plasmonic nanoparticles, $\mathrm{Fe}_{3} \mathrm{O}_{4} @$ Au core-shell, discrete dipole approximation

\section{INTRODUCTION}

Nanostructured materials which at least one dimension the particle size is between $1 \mathrm{~nm}$ to $100 \mathrm{~nm}$ have been attracted great attention of scientists due to their properties including optical, magnetic, specific heat, melting point, surface activities, chemical and biological properties are highly size dependent compared to their bulk counterparts. Also, iron oxide nanomaterial of magnetite $\left(\mathrm{Fe}_{3} \mathrm{O}_{4}\right)$ which its particle size smaller than $20 \mathrm{~nm}$ can be considered in the range of a single domain and exhibits a superparamagnetic property $^{1-2}$. A magnetic resonance imaging (MRI), medical imaging technique using a strong magnetic field and radio waves for body diagnosis, is a major application ${ }^{2-3}$. Moreover, typical metal nanoparticles (NPs) of gold (Au) and silver (Ag) have great attracted in many application $\mathrm{s}^{4-8}$ due to exhibiting of surface plasmon (SP) phenomena and optical activity. 
$\mathrm{SP}$ is electromagnetic (EM) waves coupled to the collective oscillations of the conduction electron in an interface between two media with permittivities with opposite sign such as between metal and dielectric. It can be either propagating called surface plasmon polaritons (SPPs) or propagating surface plasmons, in the planar bulk metal surface or localized surface plasmon (LSP), in the case of NPs. At certainly excited photon energy, the collective oscillations of this electron interact the most efficiently with the incident photon, result in a maximum light absorption. This is defined as localized surface plasmon resonance (LSPR). However, nanomaterials consisting of single component usually contain only one unique property of one active in gradient. Therefore, the intense research in the development of nanoparticles (NPs) that combine multiple functions or properties not obtainable in individual materials is motivated by the scientists to obtain new materials in order to further many applications. Also, nanoparticles composed of a $\mathrm{Fe}_{3} \mathrm{O}_{4}$ core, well known as magnetic properties, and continuous plasmonic (Au or Ag) shell layers, non-magnetic material but shown SP properties, for example, are of increasing interest for biomedicine application ${ }^{\text {9-12. }}$. This article the only optical absorption property in multicomponent $\mathrm{Fe}_{3} \mathrm{O}_{4} @ \mathrm{Au}$ core-shell nanoparticle embedded in water will be theoretically investigated. The surface plasmon resonance was indicated that it can be enhanced and tuned by adjusting the inter-particle distance and number of particles in the particle-chain. This study will contribute to better understanding about the optical properties which should be obtained from the construction or synthesis the $\mathrm{Fe}_{3} \mathrm{O}_{4} @$ Au core-shell bifunctional nanostructures for the associated applications.

\section{EXPERIMENTAL}

\section{Computational details}

The optical absorption efficiencies of the composite $\mathrm{Fe}_{3} \mathrm{O}_{4} @ \mathrm{Au}$ core-shell nanoparticle were calculated based on the DDA theory ${ }^{13}$. This theory, the object in arbitrary shape is replaced with an assembly of $N$ point dipoles in which the polarizability and positions are specified as $\alpha_{i}$ and $r_{i}$, respectively. The polarization induced $\mathbf{P}_{\mathbf{i}}$ in each particle in the presence of an applied field is then described by Eq. (1):

$\overrightarrow{\mathrm{P}}_{\mathrm{i}}=\alpha_{i} \overrightarrow{\mathrm{E}}_{\mathrm{loc}}\left(\overrightarrow{\mathrm{r}}_{i}\right)$

where $\mathbf{E}_{\text {loc }}$ is the sum of the incident field and the contribution from all other $\mathrm{N}-1$ dipoles. When this polarization is obtained, the absorption, scattering and extinction cross sections of light can be calculated by Eqs. (2)- (4):

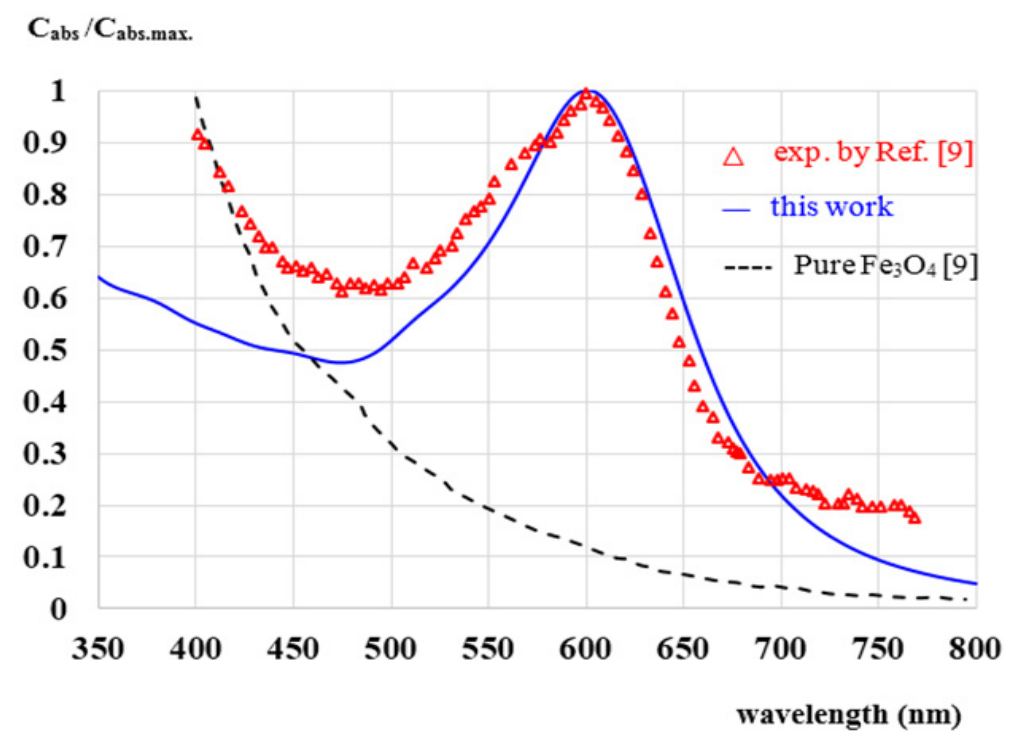

Fig. 1: Comparison between theoretical calculation and experimentally obtained UV-Visible spectrum (adapted from Ref. [$\left.{ }^{\star}\right]$ ) for a particle with an $18 \mathrm{~nm} \mathrm{Fe} \mathrm{O}_{4}$ core and $5 \mathrm{~nm}$ thick gold shell in water 


$$
\begin{aligned}
& C_{e x t}=\frac{4 \pi k}{\left|E_{0}^{2}\right|} \sum_{i=1}^{N} \operatorname{Im}\left(\overrightarrow{\mathrm{E}}_{i n c, i}^{*} \cdot \overrightarrow{\mathrm{P}}_{\mathrm{i}}\right), \\
& C_{a b s}=\frac{4 \pi k}{\left|E_{0}^{2}\right|} \sum_{i=1}^{N}\left\{\operatorname{Im}\left[\overrightarrow{\mathrm{P}}_{\mathrm{i}} \cdot\left(\alpha_{i}^{-1}\right)^{*} \overrightarrow{\mathrm{P}}_{i}^{*}\right]-\frac{2}{3} k^{3}\left|\overrightarrow{\mathrm{P}}_{i}\right|^{2}\right\}, \\
& C_{s c a}=C_{e x t}-C_{a b s} .
\end{aligned}
$$

Equations (2) and (3), '*' symbol stands for the conjugate of a complex variable, $k$ is the wave number and $E_{0}$ is the amplitude of incident electric field. And scattering, absorption and extinction efficiencies can be obtained by divided the optical cross section by their cross section area of the particle. For the composite nanoparticle assumed to be spherical, with a $\mathrm{Fe}_{3} \mathrm{O}_{4}$ core $\left(\varepsilon_{c}\right)$ of radius $b$, surrounded by a shell of gold $\left(\varepsilon_{b}\right)$ to outer radius $a$, much less than the wavelength of light being considered. The composite particle's dielectric function $\varepsilon_{s}$ is found to be ${ }^{14}$

$\varepsilon_{s}=\varepsilon_{\mathrm{b}} \frac{1+2 \beta_{c}}{1-\beta_{c}}, \beta_{c}=f_{c} \frac{\varepsilon_{\mathrm{c}}-\varepsilon_{\mathrm{b}}}{\varepsilon_{\mathrm{c}}+2 \varepsilon_{\mathrm{b}}}$

where $f_{c}=(b / c)^{3}$ is the internal volume fraction. Eventually, the absorption cross sections can be calculated using equation (3). In this calculation, the dielectric constants for $\mathrm{Fe}_{3} \mathrm{O}_{4}$ were taken from Ref. ${ }^{15}$. The $\mathrm{Fe}_{3} \mathrm{O}_{4} @$ Au core-shell nanoparticle size was taken from Lim and co-workers ${ }^{9}$. The dielectric constants for metal NPs were extracted from the experimental results in Ref. ${ }^{16}$. The dielectric constant of water which was used as the surrounding medium due is 1.77 .

\section{RESULTS AND DISCUSSION}

The calculated absorbance spectra of bimetallic nanoparticles (NPs) of $\mathrm{Fe}_{3} \mathrm{O}_{4} @ \mathrm{Au}$ coreshell NP with the $18 \mathrm{~nm} \mathrm{Fe} \mathrm{O}_{4}$ core of size coated by $5 \mathrm{~nm}$ Au layer thick embedded in water are presented. First, in Fig. 1, we show the absorbance spectra of $\mathrm{Fe}_{3} \mathrm{O}_{4} @ \mathrm{Au}$ core-shell NP in the wavelength range between $350 \mathrm{~nm}$ to $800 \mathrm{~nm}$ compared with the experimental results, $\mathrm{Fe}_{3} \mathrm{O}_{4} @$ Au core-shell NP with the same condition and pure $\mathrm{Fe}_{3} \mathrm{O}_{4} \mathrm{NP}$ (adapted from ref. ${ }^{9}$ ). Our calculations found that the LSPR peak position was agreement with the experimental data. The peak position exhibited at $\sim 602 \mathrm{~nm}$ which is in the visible wavelength region of electromagnetic (EM) wave. On the other hand, absorption peak for the single nanomaterial of pure $\mathrm{Fe}_{3} \mathrm{O}_{4}{ }^{9}$ does not obviously show any absorption in the visible region (350-800 nm). Later, in Figs. 2 and 3, the absorption efficiency of $\mathrm{Fe}_{3} \mathrm{O}_{4} @ \mathrm{Au}$ core-shell NP size and

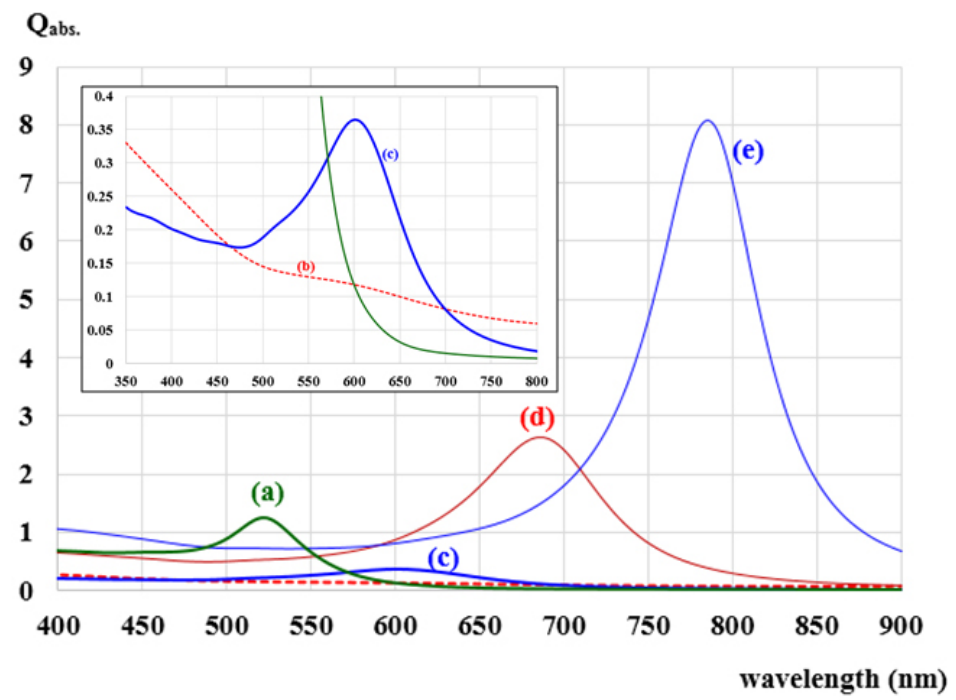

Fig. 2: Calculated of absorption efficiencies for (a) Au nanoparticle, (b) pure $\mathrm{Fe}_{3} \mathrm{O}_{4}$ nanoparticle and (c)-(e) being particles with an $18 \mathrm{~nm} \mathrm{Fe} \mathrm{O}_{4}$ core and $5 \mathrm{~nm}$ thick gold shell with varying number of particles of (c) 1 particle, (d) 2 particles and (e) 3 particles. The particles axis is ] perpendicular propagation but parallel to the polarization direction of light 
inter-particles distance were fixed, but the number of particles was varied between two particles to four particles. Fig. 2 show the absorption efficiency of the $\mathrm{Fe}_{3} \mathrm{O}_{4} @ \mathrm{Au}$ core-shell NPs aligned perpendicular to the propagation direction and parallel to the polarization direction of light. On the other hand in
Fig. 3, the $\mathrm{Fe}_{3} \mathrm{O}_{4} @$ Au core-shell NPs axis was aligned perpendicular to both the propagation direction and the polarization direction of light. First observation, by compared with the pure $\mathrm{Fe}_{3} \mathrm{O}_{4} \mathrm{NP}$ that was not obviously found the LSPR peaks, the absorption efficiency can be enhanced after coating plasmonic

Qabs.

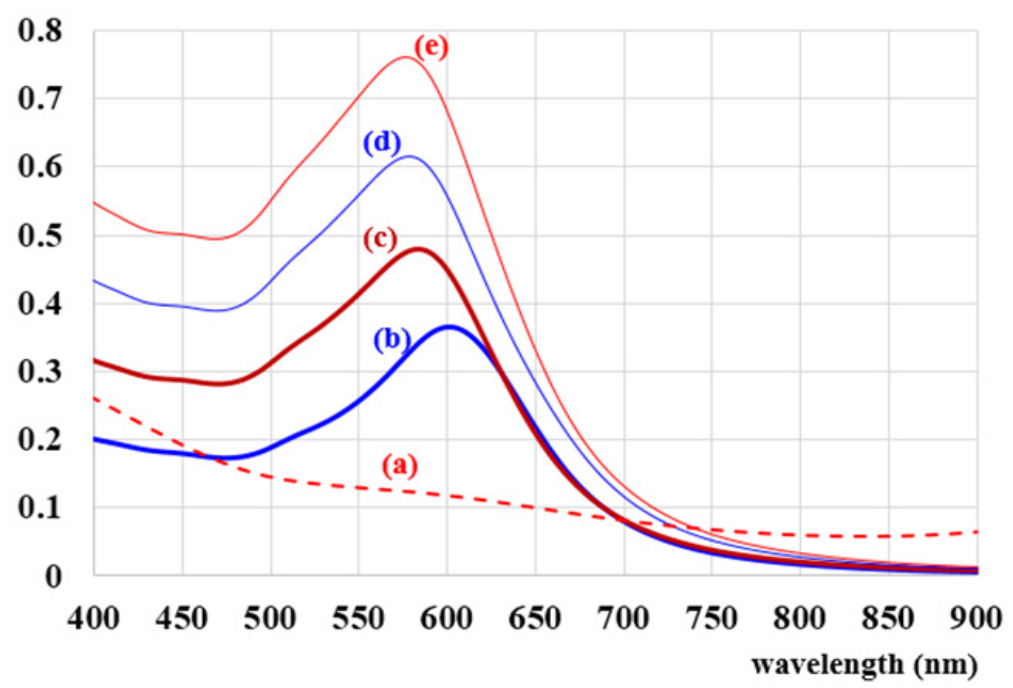

Fig. 3: calculated of absorption efficiencies for (a) pure $\mathrm{Fe}_{3} \mathrm{O}_{4}$ nanoparticle and (b)-(e) being particles with an $18 \mathrm{~nm} \mathrm{Fe} \mathrm{O}_{4}$ core and $5 \mathrm{~nm}$ thick gold shell with varying number of particles of (b) 1 particle, (c) 2 particles, (d) 3 particles and (e) 4 particles. The particles axis is perpendicular to both the polarization and propagation direction of light

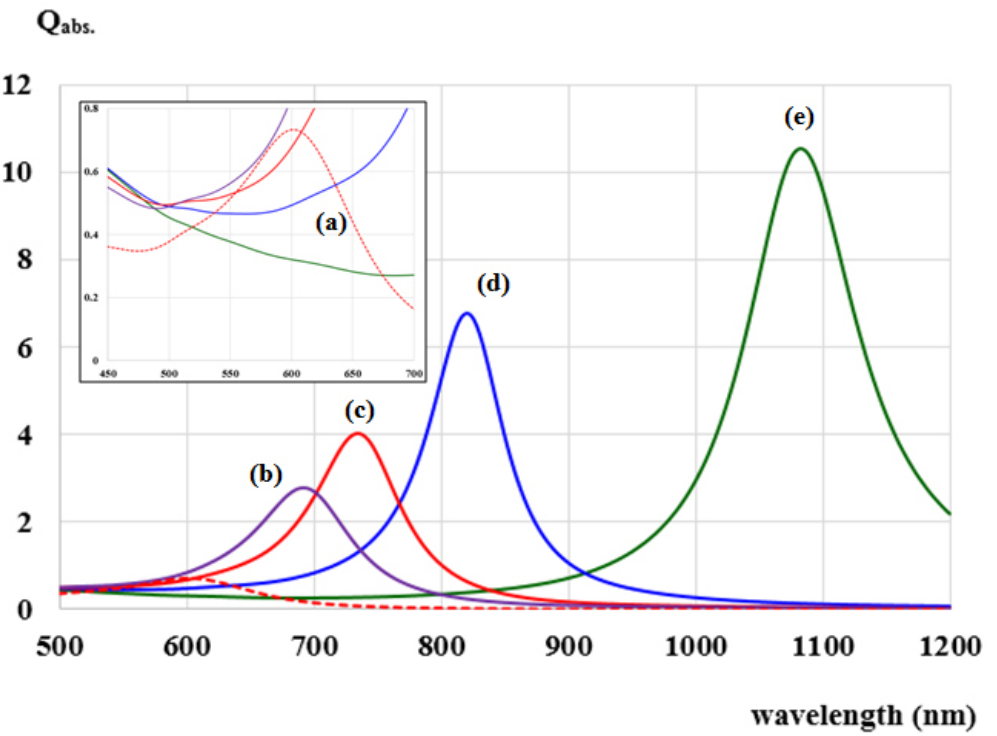

Fig. 4: Calculated absorption efficiencies in dependence of excited wavelength for two particles of an $18 \mathrm{~nm} \mathrm{Fe} \mathrm{O}_{4}$ core and $5 \mathrm{~nm}$ thick gold shell with varying the inter-particles distance, (a) 100 $\mathrm{nm}$, (b) $15 \mathrm{~nm}$, (c) $14 \mathrm{~nm}$, (d) $13 \mathrm{~nm}$ and (e) $12 \mathrm{~nm}$, aligned on x-axis 


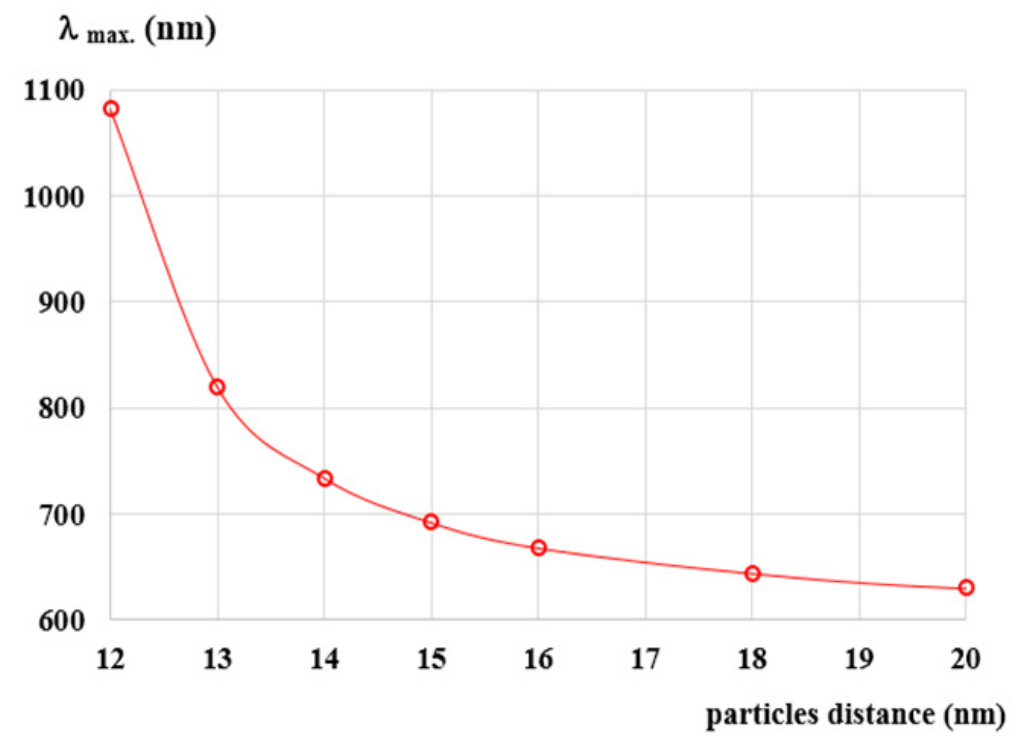

Fig. 5: Absorption peak position as function of the distance between two $\mathrm{Fe}_{3} \mathrm{O}_{4} @ \mathrm{Au}$ core-shell particles related to Fig. 4

particle of Au nanolayer on the $\mathrm{Fe}_{3} \mathrm{O}_{4}$ core. In addition for in Fig. 2, it was observed that the increasing of the number particles from 1 particle to 3 particles the LSPR peak position shifts to the red light in visible region of EM wave compared with Au spectrum (LSPR peak position at $\sim 520 \mathrm{~nm}$ ). The LSPR peak positions were strongly observed at $602 \mathrm{~nm}, \sim 686$ $\mathrm{nm}$ and $\sim 786 \mathrm{~nm}$ for nanostructured of 1 particle, 2 particles, and 3 particles, respectively. Whereas aligning particles axis perpendicular to both the propagation direction and the polarization direction of light, blue-shifted was observed as seen in Fig. 3. Last calculation, shown in Fig. 4, the absorption spectra obtained from the closely two $\mathrm{Fe}_{3} \mathrm{O}_{4} @ \mathrm{Au}$ core-shell NPs were calculated. In the calculation, these particles (fixed particles size) were aligned on the $\mathrm{x}$-axis perpendicular to the propagation direction and parallel to the polarization direction of light. The inter-particles distance was varied between $12 \mathrm{~nm}$ to $100 \mathrm{~nm}$. The obtained results were observed that the LSPR peak position was found at $\sim 1080 \mathrm{~nm}$ for $12 \mathrm{~nm}$ of particles distance and shifts to shorter wavelength of $820 \mathrm{~nm}, \sim 734 \mathrm{~nm}, \sim 692$ and $\sim 602 \mathrm{~nm}$ as the inter-particles distance changed to $13 \mathrm{~nm}, 14$ $\mathrm{nm}, 15 \mathrm{~nm}$ and $100 \mathrm{~nm}$, respectively. And it is clearly in Fig. 5 that shows the LSPR peak as a function of the inter-particles distance. It was found that the absorption peak position shifts to shorter wavelength nonlinearly with exponential-like decrease function.
The shifted resonance peak position can be qualitative explained ${ }^{17}$ that first due to the object in arbitrary shape in DDA model is replaced with an assembly of $N$ point dipoles. Therefore, the aligning particles axis parallel to the polarized light direction the plasma electron distributions of all particles is therefore perturbed with the weakening of the repulsive forces of the field. This effect leads to a correspondingly lower resonance frequency or longer wavelength as seen in the simulated results Figs. 2. In contrast, when the excited electric field incidents normal to the longer particles axis the charge distributions of each particle acts cooperatively to enhance the repulsive action in all particles, thus decreasing the resonance wavelength as seen in Figs. 3. The second explanation is the plasmon modes coupling theory ${ }^{18}$ which two distinct plasmon modes of an outer-shell surface sphere mode and an inner-shell surface cavity mode of Au shell are produced. Therefore, the splitting into two new modes of higher frequency plasmon mode and lower frequency plasmon mode is induced. This phenomenon influences the changing of LSPR peak position. It is well known that the spectral location of the LSPR is sensitive to the change of the environmental refractive index. Consequently, the high refractive index, $\sim 3-5$ in the visible region, the effect of $\mathrm{Fe}_{3} \mathrm{O}_{4}$ on the SPR frequency is large, leading to the enhancement of absorption spectra. 
The obtained results were found that it should be possible to shift the LSPRs frequency into the nearinfrared region (NIR) of the spectrum by increase the number particle and inter-particles distance as seen in Fig. 2 and Fig. 3, respectively.

\section{CONCLUSION}

In summary, the optical absorption property of the $\mathrm{Fe}_{3} \mathrm{O}_{4} @ \mathrm{Au}$ core-shell NPs with varying their number particle, inter-particle distance and direction of polarization and propagation of incident light were studied using the DDA method. The results found that the coating of $\mathrm{Au}$ nanolayer on a $\mathrm{Fe}_{3} \mathrm{O}_{4}$ spherical nanoparticle in the water which is the main component solution in the human body shows strongly LSPR induced. Moreover, the results revealed that the resonance peak of LSPR can be tuned over wavelength from the visible to the near-infrared region of the spectrum. Thus, the basic results of this study might be used for basic fabricating the more performance specific applications like a drug in life science.

\section{ACKNOWLEDGMENTS}

The authors are grateful to the Faculty of Applied Science and Engineering, Khon Kaen University for all facilities supported. This work was partially supported by the Nanotechnology Center (NANOTEC), NSTDA, Ministry of Science and Technology, Thailand through its Center of Excellence Network program.

\section{REFERENCES}

1. Goya, G. F.; Berquo, T. S.; Fonseca, F. C. J Appl. Phys. 2003, 94, 3520-3528

2. Sheng-Nan, S.; Chao, W.; Zan-Zan, Z.; YangLong, H.; Venkatraman, S. S.; Zhi-Chuan, X. Chin. Phys. B 2014, 23, 037503

3. Arbab, A. S.; Bashaw, L. A.; Miller, B. R.; Jordan, E. K.; Lewis, B. K.; Kalish H.; Frank, J. A. Radiology 2003, 229, 838-846

4. Khlebtsov, N. G.; Dykman, L. D. J. Quant. Spectrosc. Radiat. Transf. 2010, 111, 1-35

5. Liu, F.; Xie, W.; Xu, Q.; Liu, Y.; Cui, K.; Feng, X.; Zhang, W; Huang, Y. IEEE Photonics J. 2013, 5, 8400509

6. Cheng, C.-E.; Pei, Z.; Hsu, C.-C.; Chang, C.-S.; Chien, F. S.-S. Sol. Energy Mater. Sol. Cells 2014, 121, 80-84

7. Notarianni, M.; Vernon, K.; Chou, A.; Aljada, M.; Liu, J.; Motta, N. Sol. Energy 2014, 106, 23-37

8. Tang, M.; Zhu, W.; Sun, L.; Yu, J.; Qian, B.; Xiao, T. Synth. Met. 2015, 199, 69-73

9. Lim, J.; Eggeman, A.; Lanni, F.; Tilton, R. D.; Majetich, S. A. Adv. Mater. 2008, 20, 1721-1726

10
98-113

11. Banerjee, M.; Sharma, S; Chattopadhyay, A; Ghosh, S. S. Nanoscale 2011, 3, 51205125

12. Laura Rodriguez-Arco,L.; Rodriguez, I. A.; Carriel, V.; Bonhome-Espinosa, A. B.; Campos, F.; Kuzhir, P.; Duranab, J. D. G.; Lopez-Lopez, M. T. Nanoscale 2016, 8, 81388150

13. Purcell, E.M.; Pennypacker, C. R. Astrophys J. 1973, 186, 705-714

14. Dani, R. K.; Wang, H.; Bossmann, S. H.; Wysin, G.; Chikan, V. J. Chem. Phys. 2011, 135, 224502.

15. Fontijn, W.F.J.; van der Zaag, P.J.; Devillers, M.A.C.; Brabers, V.A.M.; Metselaar, R. Phys. Rev. B 1997, 56, 5432-5442

16. Johnson, P. B.; Christy R. W. Phys. Rev. B. 1972, 6, 4370-4379.

17. Rechberger, W.; Hohenau, A.; Leitner, A.; Krenn, J. R.; Lamprecht, B.; Aussenegg, F. R. Opt. Commun. 2003, 220, 137-141

18. Prodan, E.; Nordlander, P. J. Chem. Phys. 2004, 120, 5444-5454. 